\title{
La Adherencia Terapéutica en el Tratamiento del Tabaquismo
}

\section{Therapeutic Adherence in Smoking Therapy}

\author{
María Salvador Manzano \\ Sociedad Española de Especialistas en Tabaquismo
}

\author{
Francisco Javier Ayesta Ayesta \\ Universidad de Cantabria
}

\begin{abstract}
Resumen. La adherencia terapéutica es un concepto complejo y multidimensional. El porcentaje de adherencia a los tratamientos que suponen un cambio en los hábitos de vida, como es dejar de fumar, es más bajo que en otros trastornos, lo cual da lugar a consecuencias clínicas, psicológicas y económicas importantes. El presente trabajo pretende hacer un repaso por los factores relacionados con la adherencia terapéutica en el tratamiento del tabaquismo. Las estrategias para aumentar la adherencia terapéutica implican la elección adecuada del régimen terapéutico, conocer las características del fumador, derribar las barreras organizativas del sistema sanitario y preparar al profesional de la salud para conseguir desarrollar habilidades para una mejor comunicación con el paciente.

Palabras clave: adherencia terapéutica, tratamiento del tabaquismo, régimen terapéutico, barreras organizativas.
\end{abstract}

\begin{abstract}
Therapeutic adherence is a complex and multidimensional concept. The percentage of adherence to treatments involving a change in lifestyle, such as quitting smoking, is lower than in other disorders, a fact which has relevant clinical, psychological and economic consequences. This paper aims to review the associated factors with adherence to therapy in smoking treatment. Strategies to enhance therapeutic adherence involve the adequate choice of treatment, to know the smoker's characteristics, breaking down organizational barriers in health system and the training of health professionals in communication skills with patients.
\end{abstract}

Key words: therapeutic adherence, smoking therapy, treatment, organizational barriers.

\section{Introducción}

Múltiples factores pueden influir en la eficacia de un tratamiento: el trastorno al que nos enfrentemos, las características del paciente, las habilidades del terapeuta, el tipo de intervención que se lleve a cabo, etc. Además de todos estos, existe un factor clave: difícilmente un tratamiento podrá ser eficaz si el paciente no lo sigue o no lo cumple.

La adherencia de los fumadores a los tratamientos para dejar de fumar no es una cuestión de "todo o

La correspondencia sobre este artículo puede dirigirse a María Salvador Manzano, e-mail: maria.salvador.manzano@gmail.com nada". Podría hablarse de un continuo en el grado de adherencia: puede que asista a las citas, pero no tome la medicación prescrita; que se ponga bien los parches de nicotina, pero los deje antes de tiempo; que comience una terapia psicológica en grupo, pero no entrene las estrategias para controlar las deseos de fumar, etc.

En la literatura se han utilizado varios conceptos para señalar que una persona sigue un tratamiento. Haynes (1979) definió cumplimiento (compliance) como "el grado en que la conducta de un paciente, en relación con la toma de medicación, el seguimiento de una dieta o la modificación de hábitos de vida, coincide con las instrucciones proporcionadas 
por el médico o personal sanitario". Este concepto ha sido criticado porque se concede al paciente un rol pasivo, limitándose a seguir o no las instrucciones del terapeuta.

Actualmente, se considera al paciente agente activo de su propio proceso terapéutico. Esto es especialmente importante en trastornos que implican una modificación en los hábitos de la vida cotidiana: alimentarse saludablemente, seguir un plan de ejercicio físico o dejar de fumar. Desde esta perspectiva se prefiere usar el término adherencia (adherence), definido como "una implicación activa y voluntaria del paciente en un curso de comportamiento aceptado de mutuo acuerdo, cuyo fin es producir un resultado terapéutico deseado" (DiMatteo y DiNicola, 1982).

Mantener la adherencia de los fumadores a los tratamientos de cesación requiere un manejo terapéutico especial, ya que -al igual que ocurre con otras substancias adictivas- en el proceso de dejar de fumar la motivación puede fluctuar, las situaciones de riesgo pueden no ser adecuadamente valoradas y los consumos puntuales pueden dar lugar a recaídas.

La falta de adherencia a los tratamientos tiene repercusiones clínicas y económicas. A nivel clínico, que alguien no se adhiera al tratamiento, supone desaprovechar los beneficios que éste le podría generar ayudándole a dejar de fumar, lo que incide negativamente en su salud; además, al aumentar la posibilidad de recaída, la falta de adherencia puede terminar generando expectativas negativas respecto a la eficacia del tratamiento y respecto a las posibilidades de conseguir la cesación en el futuro. A nivel económico, los tratamientos que no se acaban cumpliendo suponen una pérdida de tiempo y/o de dinero para el propio paciente, para los terapeutas y para la entidad que asuma los gastos.

Identificar las variables predictoras de éxito de una intervención ahorra y polariza esfuerzos en el abordaje de cualquier trastorno. En el caso del consumo de tabaco, la adherencia al tratamiento, junto con otros factores como la ausencia de depresión, se ha identificado sistemáticamente como una variable predictora del mantenimiento de la abstinencia a largo plazo (Crucelaegui et al., 2004; López y Gil, 2001; Llambí et al., 2008).

La adherencia terapéutica es un concepto complejo, difícil de objetivar y por tanto de analizar. En este artículo se pretende aclarar qué conductas suelen estar implicadas en la falta de adherencia a los tratamientos en tabaquismo, qué factores se han asociado a ésta tradicionalmente y qué estrategias pueden favorecer una mayor adherencia a los tratamientos por parte de las personas que desean dejar de fumar.

\section{Delimitación de qué implica la adherencia}

No es fácil cuantificar inequívocamente cuántos pacientes se adhieren a los tratamientos. Como se ha señalado anteriormente, la adherencia o la falta de ella no es algo dicotómico. Otro motivo es la dificultad de encontrar instrumentos fiables para medir este factor (Bonilla, 2007): suelen utilizarse medidas indirectas, como la entrevista, donde el paciente informa de su comportamiento, lo cual no está libre de sesgos, ya que la deseabilidad social puede hacer que la persona fumadora procure no contradecir las expectativas del terapeuta y así, por ejemplo, decir que tomó la medicación tal como se le había prescrito, cuando en realidad tomó menos dosis por miedo a los efectos secundarios.

Se estima que un $40 \%$ de pacientes no se adhiere a las prescripciones terapéuticas; en el caso de las enfermedades agudas la falta de adherencia se reduce a un $20 \%$, pero en los trastornos crónicos alcanza el 45\%; si además el tratamiento conlleva cambio de hábitos o estilo de vida, como es el caso del tabaquismo, la falta de adherencia sobrepasa estos porcentajes (Haynes, et al., 2002; Rodríguez-Marín, 1995).

Diversas conductas de los pacientes pueden ser interpretadas como falta de adherencia al tratamiento: retrasos aparentemente justificados para comenzarlo, suspensión prematura del mismo, falta de asistencia a alguna consulta, experimentar resistencia al cambio de hábitos y estilo de vida para la mejora del trastorno, cumplir de forma incompleta o deficiente las indicaciones, que puede traducirse en errores de omisión, modificación de dosis, etc. (Amigo, Fernández y Pérez, 1998; Barra, 2002).

En el abordaje del consumo de tabaco, en función del tipo de tratamiento que se lleve a cabo, la falta de adherencia puede adoptar diferentes manifestaciones: 
- Tras interesarse en una terapia para dejar de fumar, muchas personas posponen la decisión cuando se les llama para comenzar; o empiezan y la abandonan tras las primeras sesiones.

- En cuanto al tratamiento farmacológico, los fumadores pueden cometer con frecuencia errores en la toma de medicación: olvidar ponerse un parche de nicotina por la mañana al levantarse, usar los chicles de nicotina masticando rápidamente buscando el alivio inmediato de la urgencia de fumar, dejar de tomar los comprimidos de bupropion o vareniclina por encontrarse mejor, etc.

- Cuando se someten a un tratamiento psicológico, a veces no se implican en las tareas: muchos terapeutas se quejan de que sus pacientes fumadores no traen los auto-registros o no ensayan las técnicas de relajación que se les enseñan; si se les recomiendan unas pautas de control de la ingesta de alimentos o de ejercicio físico, muchos no siguen las indicaciones por ser un sobreesfuerzo que no están dispuestos a soportar, etc.

La falta de adherencia no siempre debe ser contemplada como algo negativo. En ocasiones, puede ser el último - o único- mecanismo de defensa que el paciente tiene frente a consecuencias imprevistas de los tratamientos o frente a indicaciones inadecuadamente recibidas o procesadas.

En tabaco suele ocurrir, además, que los pacientes habitualmente reciben múltiples instrucciones, sugerencias o ideas que pueden facilitarles la cesación, el cambio de conducta. Sin embargo, éstas no siempre son transmitidas $-\mathrm{O}$ recibidas- de acuerdo a su importancia, lo que puede acabar ocasionando que el paciente asuma algunas de las pautas más accesorias y prescinda de alguna de las potencialmente más importantes.

No debe asumirse de manera acrítica que alguien está motivado para dejar de fumar sólo por el hecho de que siga estrictamente las indicaciones prescritas. El mejor indicador de que alguien está en disposición de aprovechar los beneficios de la terapia es que se implique activamente en ella, no como un mero "cumplidor de instrucciones", sino participando en el diseño de su plan de tratamiento. Por tanto, en vez de intentar transmitir una serie de recomen- daciones prácticas -habitualmente amplia- y esperar que sean cumplidas, es más útil analizar conjuntamente con el paciente las medidas que pueden ser más convenientes y favorecer que él mismo haga propuestas de cambio a lo largo de todo el proceso de cesación, propuestas con las que pueda comprometerse.

\section{Factores que influyen en la adherencia}

Una gran cantidad de trabajos relacionan la adhesión terapéutica y los diversos factores que pueden influir sobre ella. De una manera esquemática, éstos pueden agruparse en factores dependientes del paciente, factores dependientes de la relación profesional sanitario-paciente y otros factores, como pueden ser el tipo de trastorno, el régimen terapéutico y la organización de los servicios de salud (Amigo et al., 1998; Martín y Grau, 2004; Meichenbaum \& Turk, 1991; Rodríguez-Marín, 2004).

\section{A) Variables del paciente}

Percepción de problema. El primer requisito para que alguien que fuma se adhiera a una terapia de cesación es percibir y aceptar que tiene un problema. El hecho de implicarse en un proceso de deshabituación tabáquica, de estar en condiciones de tolerar mejor los esfuerzos y dificultades que este proceso conlleva, se ve facilitado por las siguientes tres percepciones por parte de los pacientes:

1. Percepción de que fumar le genera inconvenientes que no le compensa asumir.

2. Percepción de que el problema es solucionable.

3. Percepción de que en su caso concreto le va a ser útil la ayuda profesional que se le preste para el abandono del consumo.

Estas afirmaciones se derivan en parte del modelo de creencias de salud, propuesta de la Psicología de la Salud que refleja el interés en identificar las variables que subyacen a las conductas saludables y que ha motivado investigaciones dirigidas a descubrir las razones que llevan a las personas a proteger su salud (Ehrenzweig, 2007). Según Becker (1979) este modelo presenta capacidad explicativa y utilidad 
para promocionar el seguimiento de las prescripciones de salud. Percibir que estás expuesto a una grave enfermedad y ver las ventajas de seguir el tratamiento que trata de paliarla o curarla, se ha relacionado con la adhesión a corto plazo (Amigo et al., 1998).

Por el propio concepto de riesgo, la probabilidad de llegar a padecer enfermedades graves derivadas del consumo de tabaco no es absoluta; además, en el caso del tabaco, la mayoría de los riesgos son diferidos, se producen a medio-largo plazo. Además, quienes fuman pueden presentar creencias falsas o ideas distorsionadas sobre los riesgos para la salud que genera el tabaco, el proceso de dejar de fumar o también sobre las implicaciones del tratamiento. Son frecuentes ideas como "fumar poco no hace daño", "a mí no me va a tocar", "si siento ganas de fumar durante el tratamiento, es que no me está funcionando", "si ya me encuentro bien, debería dejar la medicación", "los medicamentos van a generarme la misma dependencia que el tabaco", etc. Las creencias falsas que impliquen una menor vulnerabilidad a la toxicidad del tabaco, una menor valoración de los riesgos o una idea distorsionada sobre el curso del tratamiento, podrían derivar en el abandono prematuro de éste, afectando con ello a la adherencia. Es por esto que en un tratamiento para dejar de fumar es fundamental identificar las falsas creencias de la persona fumadora y tratar de reestructurarlas.

Sin embargo, el modelo de creencias de salud no parece capaz de explicar totalmente la conducta de adherencia. Así, por ejemplo, un estudio realizado con pacientes con cáncer concluyó que a pesar de que el cáncer es considerado una enfermedad grave, un porcentaje de pacientes refirieron no haber seguido el tratamiento de manera adecuada (Ehrenzweig, 2007), lo que implica que hay más variables implicadas.

Ambivalencias. La motivación de las personas que deciden dejar de fumar va a fluctuar en el tiempo. Se habla del conflicto de querer y no querer: la ambivalencia (Miller \& Rollnick, 1999). La resistencia a dejar la conducta de fumar es previsible, ya que cualquier decisión humana presenta sus pros y sus contras y dejar de fumar no es una excepción a este respecto: por muchas que sean las ventajas, es lógico que también se presenten algunos inconvenientes, que en algunos momentos pueden ser percibidos como más relevantes o más urgentes.
Al igual que ocurre en el inicio del consumo, los beneficios de seguir fumando pueden ser bastante intangibles y no basarse en nada objetivo, sino sólo en expectativas creadas por la promoción y la publicidad. Sin embargo, el hecho de que el fumador los perciba puede dificultar el reconocimiento o descubrimiento de algunas ambivalencias.

Aunque las ambivalencias pueden ser manifiestas desde el inicio, lo más frecuente es que éstas vayan apareciendo a lo largo del proceso de cesación, a medida que: a) se van asumiendo como normales los beneficios de la cesación y se van olvidando las consecuencias negativas reales que ocasionaba el tabaco; b) no se logran algunas expectativas infundadas (y no siempre explícitamente formuladas) o c) aparecen circunstancias externas que alteran la percepción del balance (mi mejor amigo ha caído; ¿para que esforzarme si yo también acabaré cayendo?) o disminuyen los apoyos sociales (familia o amigos), que ven normal que ya no fume y no refuerzan su nueva conducta.

Conviene ayudar a los pacientes a que afronten y resuelvan sus ambivalencias. Para esto es útil objetivar el balance costes-beneficios de la cesación, intentando identificar la presencia de resistencias, creencias irreales y expectativas ilusorias, aunque también conviene abordar aquellos aspectos más relacionados con la conducta adictiva, como su impulsividad y los estímulos desencadenantes de los deseos de consumir (Ayesta y Rodríguez, 2007).

Coste percibido de dejar de fumar. Quien pretende dejar de fumar habitualmente está sano o presenta síntomas menores. Esto hace que, al dejar de fumar, pasen de estar "sanos" a estar "incómodos" con motivo de la sintomatología de abstinencia, o sientan malestar debido a la dificultad de controlar las situaciones para cuyo manejo usaban el consumo de tabaco. Esto puede hacer también menos soportables algunas reacciones adversas de la medicación que, aunque objetivamente sean leves, pueden ser más relevantes para el fumador que los beneficios que aportan. Ante la presencia de estos inconvenientes o menores gratificaciones a corto plazo, conviene ayudar al paciente a fijarse en los beneficios y objetivos que ya se están obteniendo y en los que se lograrán a medio-largo plazo. 
Auto-eficacia. Es otra variable del paciente a tener en cuenta; Bandura (1977) la definió como la convicción de la persona de ser capaz de realizar con éxito la conducta requerida para producir ciertos resultados. En parte dependen de la experiencia previa de la persona en otros campos, aunque puede ser aumentada: si una persona se ve capaz de aplicar alguna de las estrategias cognitivas que se han entrenado en un tratamiento psicológico para dejar de fumar, es más probable que las ponga en práctica y que le puedan resultar útiles.

Contexto personal. Aunque puede ser considerada como una variable externa al paciente, el contexto de éste puede también dificultar la adherencia (Rodríguez-Marín, 2004). Un ejemplo de esto serían aquellas personas que, dentro del tratamiento para dejar de fumar, se plantean cambiar alguna pauta de alimentación para no ganar mucho peso: es posible que no encuentren apoyo familiar, bien porque alguien vea peligrar sus hábitos alimenticios (miedo a que se deje de comprar dulces o determinados refrescos, etc.) o simplemente porque complique la elaboración de las comidas.

\section{B) Relación terapeuta-paciente}

La adhesión a los tratamientos por parte de los pacientes se relaciona estrechamente con la conducta del clínico y su capacidad de influencia en la conducta del paciente. De hecho, las variables relacionadas con la interacción sanitario-paciente están entre las más relevantes en la explicación del grado de adherencia terapéutica (Amigo et al., 1998; Rodríguez-Marín, 2004); en realidad, en la mayor parte de los casos son bastante indistinguibles las variables del paciente de las variables de la relación profesional sanitario-paciente, ya que éstas suelen ir encaminadas a mejorar o interactuar con aquéllas.

Actitud activa del paciente. Si se pide a un paciente que siga unas pautas contrarias a sus expectativas o que interfieran con sus rutinas, es probable que no las cumpla, máxime si su percepción de los costes de seguir el tratamiento son superiores a los supuestos beneficios del mismo. Es por tanto fundamental para mantener la adherencia, conseguir la participación del paciente en la elec- ción del plan terapéutico, favoreciendo que verbalice su compromiso (Amigo et al., 1998; Miller \& Rollnick, 1999).

Por ello, en el abordaje del consumo de tabaco, como ocurre en las otras conductas adictivas, una actitud del terapeuta de carácter motivacional aumenta la adherencia al tratamiento. Conviene evitar la confrontación con el paciente y no discutir con él ni tratar de imponerle un programa de tratamiento. Miller \& Rollnick (1999) sugieren que debemos aprender a bailar con nuestro paciente, ayudándole a avanzar en el proceso, sin olvidar que es él quien tiene que ver la necesidad de cambiar y hacer los esfuerzos necesarios.

Manejo de expectativas. Es imprescindible que quien desea dejar de fumar perciba que las estrategias de tratamiento le aportan algo, le sirven para afrontar situaciones concretas, le pueden resultar útiles. Por ello, la mejora de las expectativas del paciente respecto a la utilidad o beneficios del tratamiento es una manera clave de influir positivamente en la adherencia (Amigo et al., 1998).

Es así mismo importante que las expectativas de los pacientes sean congruentes con las consecuencias reales que se derivan del tratamiento. Por ejemplo, a la vez que se trasmite el potencial de un determinado fármaco o una determinada estrategia, suele ser necesario advertir que la finalidad del tratamiento no es la desaparición de los deseos de fumar y ayudar a no sobrevalorar la capacidad de un tratamiento para controlar estos deseos (Vogt et al., 2008).

Una de las principales causas de abandono prematuro del tratamiento farmacológico es la percepción de que fundamentalmente éste sirve para aliviar la sintomatología de abstinencia. Cuando se transmite esta percepción a los pacientes, éstos tienden a abandonar la medicación cuando creen que se encuentran bien. Conviene transmitir a los profesionales y a los pacientes que la razón fundamental por la que en tabaco se utilizan las herramientas farmacológicas es porque, usadas en la manera que se ha mostrado útil (esto es, entre 8 y 12 semanas), aumenta las tasas de cesación a largo plazo y que por ello, deben emplearse independientemente de la sensación de mejoría o de la reducción del deseo de fumar que puedan experimentar los pacientes. 
Del mismo modo, el conocimiento previo de algunos efectos no deseados de las estrategias terapéuticas (como reacciones adversas de la medicación o cierta sensación de aislamiento con algunas conductas de evitación) puede hacerlos más fácilmente identificables cuando aparecen, permitiendo no sobreestimarlos y poder enfrentarse más adecuadamente a ellos. Se ha descrito que la mención de los posibles efectos no deseados de un tratamiento no reduce la adhesión de los pacientes (Álvarez et al., 2001).

Adecuada comunicación. Esta es otra variable fundamental, que se solapa con las anteriores, de la que es cauce o transmisora. Una buena comunicación, en sus vertientes informativa y persuasiva, es una variable relevante en la adherencia terapéutica (Rodríguez-Marín, 2004). A la hora de informar hay que tener en cuenta la capacidad de procesamiento de los pacientes: se ha descrito que los pacientes sólo recuerdan el $50 \%$ de la información que reciben y que, además, parte de las instrucciones recibidas son recordadas erróneamente (Godoy, SánchezHuete y Muela, 1994); los consejos e instrucciones se olvidan más que otro tipo de información (Rodríguez-Marín, 2004). Conviene por tanto adaptarse a las características de los pacientes y cerciorarse de que asimilan adecuadamente las diversas informaciones necesarias: los riesgos del consumo, los beneficios de la cesación, la utilidad y la limitación de las diversas estrategias o herramientas terapéuticas, así como las características generales y particulares de todo proceso de deshabituación.

Frecuentemente los pacientes no se adhieren a determinadas pautas terapéuticas porque no perciben la importancia real que puedan tener. Conseguir transmitir esta importancia es tarea y responsabilidad del profesional. A ello ayuda la actitud empática por parte del terapeuta, la cual no sólo se relaciona con una mejor adherencia al tratamiento, sino que también es en sí un factor fundamental que facilita el cambio terapéutico (Rogers, 1957).

\section{C) Otros factores}

Características del trastorno. Diversos estudios han mostrado que la adherencia se relaciona en parte con las características del trastorno: cuando éste se manifiesta con síntomas de inicio agudo, fácilmente reconocibles y que molestan al paciente, es más probable la adhesión al tratamiento; sin embargo, cuando los síntomas son prácticamente inexistentes, poco claros o se mantienen constantes durante tiempo de manera que el paciente se adapta a ellos, la adherencia disminuye (Amigo et al., 1998).

En el caso del tabaco, los perjuicios no son siempre evidentes: el problema fundamental es que el consumo de tabaco, de cigarrillos sobre todo, es un factor de riesgo de enfermedad grave y que ésta puede debutar sin presentar sintomatología previa. Además, muchos de los síntomas asociados al consumo de tabaco (cansancio, tos matutina, modificación del timbre de voz, menor resistencia al esfuerzo, dificultad para percibir los olores y sabores, etc.) van apareciendo paulatinamente, lo que hace que la persona vaya acostumbrándose a ellos. Del mismo modo, al haberse ido asociando por repetición a diversas circunstancias de la vida del fumador, ya no le resultan extraños determinados comportamientos, propios de las conductas adictivas, como ir a comprar tabaco a cualquier hora que se acabe, planificar actividades de ocio de acuerdo a la posibilidad de consumo o dosificar -consciente o inconscientemente- el consumo, no en virtud de la apetencia.

Por estos motivos, mientras que la funcionalidad del consumo de tabaco suele ser más o menos evidente, la funcionalidad de los esfuerzos de cesación puede no serlo tanto y dificultar la adherencia a los tratamientos.

Características del régimen terapéutico. Diversos estudios han encontrado que los pacientes tienden a adherirse menos a aquellos regímenes de medicación que requieren distintas dosis a lo largo del día; también se observa que, a medida que se alargan los tratamientos, es más probable la falta de adherencia a los mismos (Sánchez, 2006). En el caso del tabaco, tomar diferentes pautas diarias requiere una mayor dedicación por parte del fumador y, por tanto, un mayor esfuerzo. En general, asociar las tomas a las comidas principales tiende a hacer menos probables los fallos producidos por olvidos.

Es importante también tener en cuenta el balance de costes y beneficios de adherirse a los tratamien- 
tos. En el caso del tabaco, los costes pueden ser diversos (economía, esfuerzo, etc.) mientras que los beneficios suelen estar más asociados a la desaparición de síntomas (Rodríguez-Marín, 2004). En las conductas preventivas, como dejar de fumar, el riesgo que se pretende evitar o la enfermedad que se pretende prevenir rara vez es contingente con la noadherencia; es decir, que el abandono del tratamiento habitualmente no supone a corto plazo la aparición de una enfermedad grave, ni tan siquiera de un síntoma molesto. Cuando el tratamiento permite el alivio o la eliminación del malestar que produce el trastorno, la adhesión podría fortalecerse por reforzamiento negativo (Amigo et al., 1998). Sin embargo, en tabaco el consumo rara vez se asocia directamente con malestar.

Entre los costes de la conducta de cesación se encuentra el coste de la medicación (no es lo mismo pagar por disfrutar que pagar por no hacerlo) y la aparición de efectos indeseados bien sea debidos al proceso de cesación o a la propia medicación. En tabaco puede ocurrir que las consecuencias de seguir un tratamiento puedan ser más punitivas (al menos, a corto plazo) que los síntomas del propio trastorno (que puede no dar ninguno). No es infrecuente que los fumadores refieran que mientras están dejando de fumar se encuentran peor que cuando fumaban, aunque esto es habitual en muchos procesos de cambio.

Al igual que en toda conducta, los efectos del tratamiento sobre el estilo de vida de un paciente actúan como potenciales condiciones reforzantes o aversivas: las consecuencias son importantes para perpetuar una conducta o no (Amigo et al.,1998). Cuando el tratamiento provoca la pérdida de gratificaciones en la vida cotidiana de un paciente, se reduce la probabilidad de que éste se adhiera al mismo, por un proceso de castigo negativo. En el proceso de dejar de fumar muchos fumadores prefieren o deben evitar al principio situaciones asociadas al consumo de tabaco, frecuentemente relacionadas con momentos de ocio con los amigos; esto, aunque sea temporal -y se ha de procurar que lo sea- supone una renuncia, algo que puede dificultar la adherencia, al igual que lo puede suponer no consumir alcohol mientras se está tomando algún medicamento.

Dejar de fumar puede suponer un cambio signifi- cativo de hábitos en aspectos fundamentales de la vida de una persona, ya que es posible que la conducta de fumar haya quedado asociada a numerosas circunstancias de la vida de un fumador: tomar café, hacer un descanso, afrontar una emoción negativa, etc. Además puede ser necesario controlar la pauta de alimentación y hacer algo de ejercicio físico para evitar una excesiva ganancia de peso, que no siempre ocurre. Dado que los regímenes terapéuticos que suponen cambios importantes en la vida cotidiana de los pacientes y requieren la incorporación de pautas de comportamiento nuevas derivan en una baja tasa de cumplimiento (Rodríguez-Marín, 2004), esto también se observa y es esperable en la cesación tabáquica.

Todo lo anterior resalta la importancia de tener en cuenta los efectos de los tratamientos que prescribimos, indicamos o aconsejamos, ya que un proceso de cesación libre - en la medida de lo posible- de efectos indeseables puede reforzar la adherencia. Así mismo, deben contemplarse las interferencias que el tratamiento genere en la vida cotidiana del fumador, para minimizar en lo posible la pérdida de gratificaciones.

\section{La organización de los servicios de salud.} Diversos factores relacionados con las características de la atención sanitaria pueden también influir en la adherencia de los pacientes a los tratamientos (Amigo et al., 1998). Respecto al abordaje del consumo de tabaco están descritos como obstáculos: la falta de formación en tabaquismo de los profesionales sanitarios, las listas de espera, los horarios de los tratamientos incompatibles con la disponibilidad del fumador, el tiempo escaso para dedicarle al paciente en la consulta, la falta de continuidad en la atención del profesional sanitario, etc.

El entrenamiento de profesionales sanitarios, haciendo énfasis en la buena comunicación entre terapeutas y pacientes aumenta la adherencia al tratamiento (Abad, 2008). Es habitual que en las instituciones que realizan tratamiento del tabaquismo existan listas de espera. Aunque esto podría servir para reafirmar al paciente en su deseo de dejar de fumar, en general, cuando una persona se interesa por incorporarse a una terapia, sería ideal que la iniciara lo antes posible para que la motivación del momento redundara positivamente en la adherencia 
al tratamiento; si se le apunta en una lista de espera prolongada, cuando se le llame para asistir, quizá se haya perdido una gran ventana de oportunidad y puede que la motivación haya disminuido. Además, los tratamientos para dejar de fumar se realizan frecuentemente en horario laboral, con lo que quien desea dejar de fumar debe faltar al trabajo para poder asistir, lo cual suele complicar más la adherencia que en otros trastornos, ya que en tabaco muy frecuentemente los fumadores están "sanos", no presentan aún una patología asociada relevante. Por otra parte, la falta de continuidad en la atención del profesional sanitario, hace que en ocasiones la intervención sea realizada por una persona y el seguimiento por otra, lo que puede influir negativamente en el vínculo terapéutico, pudiéndose resentir, por tanto, la adherencia.

\section{Estrategias para mejorar la adherencia en tabaco}

A continuación se indican una serie de estrategias que pueden ser útiles para mejorar el seguimiento de los tratamientos para dejar de fumar (Abad, 2008; Amigo et al., 1998):

- Conseguir establecer un buen vínculo terapéutico con el paciente. Esto es especialmente relevante en los procesos crónicos que conllevan un cambio de conducta y/o actitudes, como es el caso del tabaco. Se consigue mostrando interés por las peculiaridades del fumador, sus necesidades y preferencias, sus expectativas y temores, mostrando empatía y expresando confianza en el tratamiento y en el paciente, lo cual derivará en el aumento de sus expectativas positivas con respecto a sus posibilidades de dejar de fumar.

- Anticipar la falta de adhesión. Ser consciente de las dificultades con las que puede encontrarse una persona fumadora para seguir el tratamiento, permite anticiparse a ellas. Conviene identificar las posibles creencias falsas que pudiera tener e indagar qué características de su contexto (familiar, social o laboral) pueden favorecer u obstaculizar la adhesión. Es de gran ayuda instar al fumador a que busque apoyo social para afrontar las dificultades que se planteen y fuentes alternativas de gratificación, en previsión de que a lo largo del tratamiento pueda perder alguna de las que tenía.

- Conocer e incorporar como objetivos los propios del paciente (dentro de las posibilidades clínicas). Es indispensable conocer las expectativas del fumador respecto las diferentes herramientas terapéuticas que puedan ofrecérsele para así poder encauzarlas, reestructurarlas y, en la medida de lo posible, adaptarse a ellas. Si una persona cree que asistiendo a las sesiones de una terapia en grupo para dejar de fumar le va a resultar más fácil que si lo hace con un tratamiento individual, sería deseable elegir la primera opción. Sin embargo, es importante ajustar las expectativas erróneas del paciente a la realidad: si cree que en la terapia para dejar de fumar en grupo va a ser convencido para que deje de fumar, es fundamental corregir esta expectativa.

- Ofrecer información y asegurarnos de que el paciente la recibe. La información que se facilite al fumador sobre el tratamiento ha de describir la conducta que se solicita, las condiciones (rutinas cotidianas) en las que ha de utilizarse, la utilidad del tratamiento, las consecuencias positivas y negativas que cabe esperar y cómo manejar los efectos secundarios si aparecen. El objetivo es conseguir que la información que se considera necesaria llegue al paciente, que la comunicación sea eficaz. Los profesionales de la salud tienden a sobreestimar la importancia del tiempo que emplean dando información al paciente y a subestimar el deseo de los pacientes para obtener información (Rodríguez, 1999). Una estrategia que ayuda al paciente a recibir la información adecuadamente es darle un folleto con la información fundamental y repasarla juntos. Conviene también sondear las posibles dudas que el fumador quizá no se atreva a preguntar. Es recomendable que las indicaciones sean específicas y operativas: por ejemplo, si dentro del tratamiento para dejar de fumar vamos a incluir el ejercicio físico para evitar el aumento de peso, no basta con decir "haga ejercicio", sino que es preferible 
decirle, “¿qué le parece si a partir de ahora sube a su casa por las escaleras al menos una vez al día?".

- Simplificar el tratamiento e individualizarlo en lo posible al estilo de vida del paciente. En los procesos crónicos la simplificación de las pautas dosificadoras mejora la adherencia (Álvarez et al., 2001). Dentro de lo clínicamente posible, es más fácil la adherencia al tratamiento farmacológico si las dosis son siempre similares y se mantiene una cierta flexibilidad con los horarios y hábitos del fumador. Si se da la indicación de "subir las escaleras de su casa al menos una vez al día" a una persona que vive en un $9^{\circ}$ piso, seguramente no la seguirá; en ese caso, puede sugerirse que use el ascensor hasta el $7^{\circ}$ piso, por ejemplo. Es importante tener en cuenta el grado de interferencia del tratamiento en la rutina diaria y contexto del paciente. Anticipando los problemas que puedan aparecer, se pueden buscar soluciones conjuntas.

- Establecer metas terapéuticas asequibles y consensuadas con el paciente. Como se ha señalado, conocer las preferencias del fumador e involucrarle activamente en su tratamiento aumenta la adherencia al mismo. Conviene planificar los objetivos terapéuticos con el paciente en función de lo que considere asequible e importante. Si un fumador desea que el inicio de la cesación sea progresivo y no desea reducir todo de golpe, puede hacerse un inicio progresivo. Si considera mejor no retirar los ceniceros de casa, mostraremos respeto a su decisión. Si en un tratamiento en grupo una persona refiere necesitar más tiempo para dar un determinado paso, compensa acompañar a esa persona con el ritmo que nos marque. A este respecto, ayuda establecer una "jerarquía de estrategias”, más o menos explícita, en la que se muestren al fumador las ventajas de seguir aquellas que son consideradas fundamentales, pero dándole margen para elegir utilizar o no otras que se consideren más secundarias.

- Entrenar las competencias requeridas al paciente. Cuando un paciente cuenta con las competencias necesarias para seguir las pautas establecidas, aumenta su autoeficacia percibi- da. Conviene confirmar que el fumador es capaz de realizar correctamente las estrategias indicadas, siendo en muchos casos necesario ensayar la conducta concreta. Rellenar un autoregistro del consumo de cigarrillos no es tarea fácil. Para que este instrumento tenga utilidad, hay que rellenarlo correctamente: para ello hay que saber identificar las circunstancias que elicitan el deseo de fumar (antecedentes) y los procesos (internos y externos) que se desencadenan una vez se ha fumado el cigarro (consecuentes); esta tarea requiere entrenamiento. Del mismo modo, es útil ensayar con algunos pacientes cómo rechazar tabaco cuando le ofrecen, cómo solicitar que no se fume en su presencia o cómo pedir ayuda a alguien de su entorno en momentos concretos de deseos intensos de consumir.

- Reforzar el seguimiento. Uno de los reforzadores más potentes son los propios efectos del tratamiento sobre la sintomatología y sobre el estado psicológico del paciente, pero esto no siempre es posible percibirlo. Cambiar conductas aprendidas conlleva siempre un esfuerzo y, por muy reforzador que sea mantenerse sin fumar, afrontar las situaciones cotidianas de la vida sin el apoyo del tabaco puede costar. Es importante reforzar que los esfuerzos por mantener la abstinencia son importantes; conviene mantener la empatía y reforzar los comportamientos de adherencia del fumador, aun cuando no haya conseguido mantenerse abstinente.

- Favorecer la accesibilidad y continuidad de la atención. En la medida de lo posible se deben intentar controlar las variables dependientes de la organización sanitaria que dificultan la adhesión. Conviene que la espera tras realizar una demanda de ayuda para dejar de fumar no se alargue desproporcionadamente. Debe procurarse que las sesiones de seguimiento sean atendidas por un mismo terapeuta, si es posible (Martín, 2006). Puede también ser útil establecer un protocolo de seguimiento, de manera que pueda llamarse a alguien que no acude a alguna cita, mostrando interés por la persona y motivándole para que continúe (Álvarez et al., 2001). 


\section{Consideraciones finales}

Muchas intervenciones terapéuticas pierden eficacia porque los pacientes no se adhieren adecuadamente a ellas. Esto es especialmente relevante en los procesos que implican cambios de conducta, como el abandono del consumo de sustancias adictivas, como el tabaco.

De una manera resumida puede decirse que alguien no se adhiere a una determinada pauta, bien porque no percibe su importancia real o porque determinados obstáculos, intrínsecos o extrínsecos, le impiden adherirse a ellas.

En cualquier caso, las habilidades -especialmente de comunicación- del terapeuta son el elemento clave para conseguir una mayor adherencia, tanto a la hora de detectar los posibles problemas como a la hora de solucionarlos.

En general, los pacientes se esfuerzan por conseguir aquellos objetivos que creen que les merece la pena conseguir y que piensan que son asequibles. Por ello, tienden a adherirse a aquellas medidas que consideran que les ayudarán a conseguir estos objetivos y/o a hacerlos más factibles.

En el caso del consumo de substancias adictivas, la cesación puede verse obstaculizada por dificultades propias del proceso de abandono, bien sean intrínsecas (como la pérdida de gratificaciones inmediatas o las ambivalencias respecto a la cesación) o extrínsecas (como un sistema de salud no diseñado para atender a personas con actividad laboral y familiar normal).

Los dos párrafos anteriores resumen las claves de cómo afrontar de la manera más adecuada la adherencia al tratamiento en los procesos de cesación tabáquica. Los profesionales sanitarios deben ayudar a sus pacientes a percibir de la manera más inequívoca posible -es decir, ajustándose a sus circunstancias personales- que:

- les merece la pena dejar de fumar.

- pueden conseguirlo.

- las estrategias y objetivos intermedios propuestos y asumidos tienen su razón de ser y son asequibles.

Esto supone transmitir información adecuada sobre el consumo de tabaco y el proceso de cesación y facilitar la eliminación de obstáculos al cambio.
Esto último implica ayudar al paciente a mostrar sus expectativas y creencias para intentar ajustarlas a la realidad del proceso de abandono, a descubrir y a afrontar sus ambivalencias respecto al mismo y transmitir lo que se espera o puede esperar de cada estrategia terapéutica.

Aun así, la clínica, además de ciencia, es un arte y las medidas dirigidas a aumentar la adherencia al tratamiento no siempre consiguen sus objetivos (Kripalani et al., 2007). Como se exponía al principio del presente artículo, en el cambio de conductas que afectan a los hábitos de la vida cotidiana el porcentaje de adherencia al tratamiento no suele ser muy elevado; Wiggers et al. (2006) lo han confirmado en estudios en fumadores.

Por ello es importante plantearse el mantenimiento de la adherencia a los tratamientos como un objetivo terapéutico más, manejando una combinación de todas las estrategias implicadas en este proceso, especialmente las que contemplan los factores psicosociales (Williams, Manias, \& Walker, 2008) para lograr mejores resultados y conseguir mantenerlos a largo plazo (Haynes et al., 2002, 2008).

\section{Referencias}

Abad, F. (2008). La formación de los médicos mejora la adherencia al tratamiento antihipertensivo. Actualidad en Farmacología y Terapéutica, 6, 40-42.

Álvarez, S., Gómez, F.J., Gallego, P. y Picón G, C. (2001). Estrategias eficaces para mejorar la adherencia a la prescripción de fármacos. Medifam [online] 11,8. Obtenido el 17 de junio de 2009 en: http://scielo.isciii.es/scielo.php? script $=$ sci_arttext\&pid $=$ S 1131-5768200100 $0800007 \&$ lng=es\&nrm=iso $>$ ISSN 1131-5768.

Amigo, I., Fernández, C. y Pérez, M. (1998). La adhesión a los tratamientos terapéuticos. En I. Amigo (Ed.), Manual de Psicología de la Salud (cap. 13, pp. 229-270). Madrid: Pirámide.

Ayesta, F. J. y Rodríguez, M. (2007) Bases biológicas, conductuales y sociales de las dependencias: tabaco y nicotina. Manual PIUFET $n^{o} 1$, Santander: Ediciones PIUFET. 
Bandura, W. (1977). Self-efficacy. Toward a unifying theory of behavioral change. Psychological Review, 84, 191-215.

Barra, E. (2002). Psicología de la Salud. Chile: Universidad de Concepción

Becker, M. (1979). Understanding patient compliance: the contributions of attitudes and other psychosocial factors. En S. Cohen (Ed.). New directions in patient compliance (pp. 1-31). Lexington, MA: Lexington Books.

Bonilla, C. P. (2007). Diseño de un instrumento para evaluar los factores que influyen en la adherencia a tratamientos, en personas que presentan factores de riesgo de enfermedad cardiovascular. Avances en Enfermería, 2, 46-55.

Crucelaegui, A., Nerín, I., Sobradiel N., Belmonte, A. Novella, P., Bernal, V. y Gargallo, P. (2004). Factores predictores de éxito a los seis meses en fumadores tratados en una unidad de tabaquismo. Prevención del Tabaquismo 6, 175-6.

DiMatteo, M. y DiNicola, D. (1982). Achieving patient compliance: the psychology of the medical practitioner's role. Nueva York. Pergamon Press.

Ehrenzweig, Y. (2007). Modelos de cognición social y adherencia terapéutica en pacientes con cáncer. Avances en Psicología Latinoamericana, 25, 721.

Godoy, J., Sánchez-Huete, J., y Muela, J. (1994). Evaluación del nivel de comunicación médicopaciente en la consulta ambulatoria. Revista de Psicología de la Salud, 6, 103-122.

Haynes, R. (1979). Introduction. En R. Haynes, D. Taylor y D. Sackett (Eds.), Compliance in health care (pp. 1-7). Baltimore: Johns Hopkins University Press.

Haynes, R.B., Ackloo, E., Sahota, N., McDonald, H.P., \& Yao, X. (2008). Interventions for enhancing medication adherente. Cochrane Database of Systematic Reviews. Issue 2. Art. No.: CD000011. DOI: 10.1002/14651858.CD000011.pub3.

Haynes, R.B., McDonald, H.P. \& Garg. A.X. (2002). Helping Patients Follow prescribed treatment. Clínical applications. The Journal of the American Medical Association, 288, 2880-83.

Kripalani, S., Yao, X. \& Haynes, R.B. (2007). Interventions to enhance medication adherence in chronic medical conditions: a systematic review. Archives of Internal Medicine, 167 (6), 540-50.

López, F. y Gil, J. (2001). Características del fumador y su relación con el abandono del tabaco en programas de intervención no aversivos. International Journal of Clinical and Health Psychology, 1, 333-351.

Llambí, M. L., Esteves, E, Blanco, M. L., Barros, M, Parodi, C y Goja, B, (2008). Factores predictores de éxito en el tratamiento del tabaquismo. Revista Médica del Uruguay, 24, 83-94.

Martín, L. (2006). Repercusiones para la salud pública de la adherencia terapéutica deficiente. Revista Cubana de Salud Pública, [online] 32,3. Obtenido el 19 de junio de 2009 en: http://bvs.sld.cu/revistas/spu/vol32_3_06/spu133 06.htm

Martín, L. y Grau, J. (2004). La investigación de la adherencia terapéutica como un problema de la Psicología de la salud. Psicología y Salud, 14, 8999.

Meichenbaum, D. y Turk, D. (1991). Cómo facilitar el seguimiento de los tratamientos terapéuticos. Bilbao: DDB.

Miller, W.R. \& Rollnick, S. (1999). La entrevista motivacional. Preparar para el cambio de conductas adictivas. Barcelona: Paidós.

Rodríguez-Marín, J. (1995). Efectos de la interacción entre el profesional sanitario y el paciente. Satisfacción del paciente. Cumplimiento de las prescripciones terapéuticas. En J. RodríguezMarín (Ed.). Psicología social de la salud (pp. 151-160). Madrid: Síntesis.

Rodríguez-Marín, J. (2004) Cumplimiento terapéutico del paciente y seguridad clínica. Monografías Humanitarias, 8,101-110.

Rodríguez, G. (1999). Fortalecimiento de la adherencia terapéutica. México: Miguel Angel Porrúa.

Rogers, R. (1957). The necessary and sufficient conditions of therapeutic personality change. Journal of Consulting Psychology, 21, 95-103.

Sánchez, N. (2006), Adherencia terapéutica en hipertensión arterial sistémica. Revista Mexicana de Enfermería Cardiológica, 14, 98-101.

Vogt, F., Hall, S. \& Marteau, T.M. (2008). Understanding why smokers do not want to use 
nicotine dependence medications to stop smoking: Qualitative and quantitative studies. Nicotine \& Tobacco Research, 10, 1405-13.

Wiggers. L. C., Smets, E. M., Oort, F. J., StormVersloot, M. N., Vermeulen, H., Van Loenen, L. B., Peters, R. J., de Haes, H. C. \& Legemate, D. A. (2006). Adherence to nicotine replacement patch therapy in cardiovascular patients. International Journal of Behavioral Medicine, 13, 79-88.

Williams, A., Manias, E. \& Walker, R. (2008). Interventions to improve medication adherence in people with multiple chronic conditions: a systematic review. Journal of Advanced Nursing, 63, 132-43.

Manuscrito recibido: 25/11/2009

Revisión recibida:10/12/2009

Manuscrito aceptado:14/12/2009 